\title{
Additional Simulation Results
}

Supplementary material for:

\section{Assessing transient carryover effects in recurrent event processes, with application to chronic health conditions}

\author{
Candemir Çı̆̆şar ${ }^{1}$ and Jerald F. Lawless ${ }^{2}$ \\ ${ }^{1}$ Women's College Hospital* and Princess Margaret Hospital ${ }^{\dagger}$ \\ ${ }^{2}$ University of Waterloo:
}

Correspondence to: Candemir Çı̆̆şar

Email: candemir.cigsar@wchospital.ca

Phone: +1 416 - 3513732 ext. 2331

Fax: +1 $416-3513746$

Address: Women's College Research Institute, 790 Bay Street, 7th Floor, Toronto, Ontario M5G 1N8, Canada

\footnotetext{
*Women's College Research Insitute, 790 Bay Street, 7th Floor, Toronto, Ontario M5G 1N8, Canada

${ }^{\dagger}$ Department of Biostatistics, 610 University Avenue, Toronto, Ontario M5G 2M9, Canada

${ }^{\ddagger}$ Department of Statistics and Actuarial Science, 200 University Avenue West, Waterloo, Ontario N2L 3G1, Canada
} 


\section{More detailed look at frailty and carryover effects}

Details of the simulation study in this section are given in the paper pp. 6-7.

\begin{tabular}{lcccc} 
& $\phi$ & $\gamma$ & $m$ & $\Delta$ \\
\hline Scenario 1 & 0.2 & 2 & 100 & 0.05 \\
Scenario 2 & 0.2 & 2 & 500 & 0.05 \\
Scenario 3 & 0.2 & 5 & 100 & 0.05 \\
Scenario 4 & 0.2 & 5 & 500 & 0.05 \\
Scenario 5 & 0.5 & 2 & 100 & 0.05 \\
Scenario 6 & 0.5 & 2 & 500 & 0.05 \\
Scenario 7 & 0.5 & 5 & 100 & 0.05 \\
Scenario 8 & 0.5 & 5 & 500 & 0.05 \\
\hline
\end{tabular}

S. 1: Scenarios with the combinations of $(\phi, \gamma, m, \Delta)$ that are used in Tables S. 2 and S. 3.

\begin{tabular}{lcccccc} 
& $\overline{\hat{\gamma}}$ & $\bar{s}(\hat{\gamma})$ & $\operatorname{Esd}(\hat{\gamma})$ & $\overline{\hat{\beta}}$ & $\bar{s}(\hat{\beta})$ & $\operatorname{Esd}(\hat{\beta})$ \\
\hline Scenario 1 & 1.960 & 0.1468 & 0.1711 & 0.169 & 0.2321 & 0.2435 \\
Scenario 2 & 1.961 & 0.0657 & 0.0722 & 0.185 & 0.1020 & 0.1062 \\
Scenario 3 & 4.764 & 0.2457 & 0.3222 & 0.208 & 0.1041 & 0.1128 \\
Scenario 4 & 4.778 & 0.1101 & 0.1430 & 0.203 & 0.0464 & 0.0486 \\
Scenario 5 & 1.904 & 0.1446 & 0.1838 & 0.409 & 0.2133 & 0.2307 \\
Scenario 6 & 1.907 & 0.0647 & 0.0835 & 0.426 & 0.0936 & 0.0986 \\
Scenario 7 & 4.448 & 0.2363 & 0.3749 & 0.460 & 0.0995 & 0.1227 \\
Scenario 8 & 4.461 & 0.1059 & 0.1614 & 0.468 & 0.0440 & 0.0552 \\
\hline
\end{tabular}

S. 2: We generated 1,000 realizations of the random effect model $\lambda_{i}\left(t \mid \mathcal{H}_{i}(t)\right)=\alpha_{i}$, where $\alpha_{i} \sim$ gamma $[$ mean $=1$, variance $=\phi]$, and then fitted the model $\gamma \exp \left(\beta Z_{i}(t)\right)$, where $Z_{i}(t)=I\left(N_{i}\left(t^{-}\right)\right) I\left(B_{i}(t) \leq \Delta\right)$. $\overline{\hat{\gamma}}$ and $\overline{\hat{\beta}}$ are the averages of the estimates $\hat{\gamma}$ and $\hat{\beta}$ and $\bar{s}(\hat{\gamma})$ and $\bar{s}(\hat{\beta})$ are the averages of the standard errors of $\hat{\gamma}$ and $\hat{\beta}$ (found by inverting the information matrix) across the 1,000 simulation runs. $\operatorname{Esd}(\hat{\gamma})$ and $\operatorname{Esd}(\hat{\beta})$ are the empirical standard deviations of $\hat{\gamma}$ and $\hat{\beta}$. 


\begin{tabular}{lccccccccc} 
& $\overline{\hat{\gamma}}$ & $\bar{s}(\hat{\gamma})$ & $\operatorname{Esd}(\hat{\gamma})$ & $\overline{\hat{\beta}}$ & $\bar{s}(\hat{\beta})$ & $\operatorname{Esd}(\hat{\beta})$ & $\overline{\hat{\phi}}$ & $\bar{s}(\hat{\phi})$ & $E s d(\hat{\phi})$ \\
\hline Scenario 1 & 1.998 & 0.1746 & 0.1783 & -0.0151 & 0.2448 & 0.2517 & 0.196 & 0.1083 & 0.1095 \\
Scenario 2 & 1.999 & 0.0783 & 0.0752 & -0.0046 & 0.1078 & 0.1099 & 0.198 & 0.0493 & 0.0506 \\
Scenario 3 & 4.995 & 0.3443 & 0.3523 & 0.0025 & 0.1108 & 0.1149 & 0.201 & 0.0616 & 0.0611 \\
Scenario 4 & 5.008 & 0.1540 & 0.1566 & -0.0011 & 0.0494 & 0.0492 & 0.198 & 0.0273 & 0.0266 \\
Scenario 5 & 1.995 & 0.2071 & 0.1981 & -0.0066 & 0.2270 & 0.2297 & 0.494 & 0.1645 & 0.1695 \\
Scenario 6 & 2.000 & 0.0934 & 0.0915 & -0.0025 & 0.0997 & 0.0979 & 0.501 & 0.0734 & 0.0725 \\
Scenario 7 & 4.988 & 0.4455 & 0.4516 & -0.0019 & 0.1081 & 0.1057 & 0.501 & 0.1083 & 0.1084 \\
Scenario 8 & 5.006 & 0.2004 & 0.1971 & 0.0016 & 0.0479 & 0.0469 & 0.503 & 0.0483 & 0.0493 \\
\hline
\end{tabular}

S. 3: We generated 1,000 realizations of the random effect model $\lambda_{i}\left(t \mid \mathcal{H}_{i}(t)\right)=\alpha_{i}$, where $\alpha_{i} \sim$ gamma $[$ mean $=1$, variance $=\phi]$, and then fitted the model $\lambda_{i}\left(t \mid \mathcal{H}_{i}(t), \alpha_{i}\right)=\alpha_{i} \gamma \exp \left(\beta Z_{i}(t)\right)$, where $Z_{i}(t)=$ $I\left(N_{i}\left(t^{-}\right)\right) I\left(B_{i}(t) \leq \Delta\right) . \overline{\hat{\gamma}}, \overline{\hat{\beta}}$ and $\overline{\hat{\phi}}$ are the averages of the estimates $\hat{\gamma}, \hat{\beta}, \hat{\phi}$ and $\bar{s}(\hat{\gamma}), \bar{s}(\hat{\beta})$ and $\bar{s}(\hat{\phi})$ are the averages of the standard errors of $\hat{\gamma}, \hat{\beta}, \hat{\phi}$ (found by inverting the information matrix) across the 1,000 simulation runs. $\operatorname{Esd}(\hat{\gamma}), \operatorname{Esd}(\hat{\beta})$ and $\operatorname{Esd}(\hat{\phi})$ are the empirical standard deviations of $\hat{\gamma}, \hat{\beta}$ and $\hat{\phi}$, respectively.

\section{Additional results for power of test statistic (3.6)}

Table S. 4 shows the results for different $\left(\Delta_{0}, e^{\beta}, m, \tau\right)$ combinations when $\phi=0.6$. A discussion of the case when $\phi=0.3$ is given in the paper in Section 4 (also see Table 4 in the paper).

\begin{tabular}{ccccc|ccc|ccc}
\hline & & \multicolumn{3}{c}{$m=20, \tau=10$} & \multicolumn{3}{c}{$m=40, \tau=5$} & \multicolumn{3}{c}{$m=40, \tau=10$} \\
\cline { 2 - 10 }$\Delta$ & $\Delta_{0}$ & $e^{\beta}=2$ & $e^{\beta}=3$ & $e^{\beta}=4$ & $e^{\beta}=2$ & $e^{\beta}=3$ & $e^{\beta}=4$ & $e^{\beta}=2$ & $e^{\beta}=3$ & $e^{\beta}=4$ \\
\hline \multirow{3}{*}{0.0202} & $\frac{2}{3} \Delta$ & 0.322 & 0.766 & 0.924 & 0.327 & 0.752 & 0.944 & 0.557 & 0.940 & 0.997 \\
& $\Delta$ & 0.566 & 0.923 & 0.991 & 0.537 & 0.937 & 0.992 & 0.846 & 0.995 & 1.000 \\
& $\frac{4}{3} \Delta$ & 0.551 & 0.938 & 0.989 & 0.553 & 0.918 & 0.996 & 0.813 & 0.999 & 1.000 \\
\hline \multirow{3}{*}{0.0513} & $\frac{2}{3} \Delta$ & 0.633 & 0.964 & 0.996 & 0.623 & 0.976 & 1.000 & 0.857 & 1.000 & 1.000 \\
& $\Delta$ & 0.871 & 0.996 & 1.000 & 0.856 & 0.999 & 1.000 & 0.981 & 1.000 & 1.000 \\
& $\frac{4}{3} \Delta$ & 0.844 & 0.998 & 0.999 & 0.837 & 0.998 & 1.000 & 0.981 & 1.000 & 1.000 \\
\hline \multirow{3}{*}{0.1054} & $\frac{2}{3} \Delta$ & 0.815 & 0.998 & 1.000 & 0.854 & 0.998 & 1.000 & 0.977 & 1.000 & 1.000 \\
& $\Delta$ & 0.966 & 1.000 & 1.000 & 0.976 & 1.000 & 1.000 & 1.000 & 1.000 & 1.000 \\
& $\frac{4}{3} \Delta$ & 0.950 & 1.000 & 1.000 & 0.957 & 1.000 & 1.000 & 0.998 & 1.000 & 1.000 \\
\hline
\end{tabular}

S. 4: Proportion of times in 1,000 samples that test statistic (3.6) exceeded its 0.05 critical value for the alternative model (4.1) under various scenarios when $\phi=0.6$. 


\section{Large $m$ and small $E\left\{N_{i}\left(\tau_{i}\right)\right\}$ case: Simulation results}

Details of the simulation study in this section are given in the paper in Section 4 (p. 14 last paragraph).

\begin{tabular}{cccccccc}
\hline$m$ & $E\left\{N_{i}\left(\tau_{i}\right)\right\}$ & $\hat{Q}_{.950}$ & $\hat{Q}_{.975}$ & $\hat{Q}_{.990}$ & $\hat{P}(S>1.645)$ & $\hat{P}(S>1.960)$ & $\hat{P}(S>2.326)$ \\
\hline \multirow{2}{*}{100} & 1 & 1.683 & 2.055 & 2.533 & 0.0538 & 0.0303 & 0.0141 \\
& 2 & 1.706 & 2.052 & 2.507 & 0.0585 & 0.0306 & 0.0136 \\
& 5 & 1.637 & 1.948 & 2.300 & 0.0487 & 0.0247 & 0.0094 \\
\hline 200 & 1 & 1.680 & 2.005 & 2.432 & 0.0536 & 0.0281 & 0.0125 \\
& 2 & 1.682 & 2.017 & 2.480 & 0.0527 & 0.0287 & 0.0135 \\
& 5 & 1.668 & 2.001 & 2.405 & 0.0527 & 0.0274 & 0.0123 \\
\hline 500 & 1 & 1.700 & 2.008 & 2.455 & 0.0557 & 0.0288 & 0.0128 \\
& 2 & 1.631 & 2.006 & 2.376 & 0.0495 & 0.0272 & 0.0115 \\
& 5 & 1.673 & 1.999 & 2.310 & 0.0527 & 0.0277 & 0.0093 \\
\hline
\end{tabular}

S. 5: $\hat{Q}_{p}$ is the empirical $p$ th quantile of $S$ in (3.6) computed from 10,000 samples when $\Delta=0.0513$. $\hat{P}\left(S>Q_{p}\right)$ is the proportion of the values of $S$ in 10,000 samples which are larger than the $p$ th quantile of a standard normal distribution. The null model $(4.1)$ has $\rho_{0}(t ; \gamma)=1, \beta=0$ and $\alpha_{i} \sim$ gamma [mean=1, variance $=0.6]$.

\begin{tabular}{cccccccc}
\hline$m$ & $E\left\{N_{i}\left(\tau_{i}\right)\right\}$ & $\hat{Q}_{.950}$ & $\hat{Q}_{.975}$ & $\hat{Q}_{.990}$ & $\hat{P}(S>1.645)$ & $\hat{P}(S>1.960)$ & $\hat{P}(S>2.326)$ \\
\hline \multirow{2}{*}{100} & 1 & 1.682 & 2.022 & 2.390 & 0.0535 & 0.0281 & 0.0116 \\
& 2 & 1.633 & 1.984 & 2.354 & 0.0482 & 0.0265 & 0.0110 \\
& 5 & 1.673 & 1.985 & 2.402 & 0.0522 & 0.0277 & 0.0123 \\
\hline 200 & 1 & 1.690 & 2.006 & 2.362 & 0.0543 & 0.0278 & 0.0109 \\
& 2 & 1.651 & 2.005 & 2.351 & 0.0508 & 0.0268 & 0.0108 \\
& 5 & 1.685 & 2.000 & 2.385 & 0.0541 & 0.0275 & 0.0116 \\
\hline 500 & 1 & 1.647 & 1.992 & 2.404 & 0.0503 & 0.0270 & 0.0113 \\
& 2 & 1.659 & 1.970 & 2.372 & 0.0513 & 0.0260 & 0.0113 \\
& 5 & 1.580 & 1.913 & 2.285 & 0.0426 & 0.0231 & 0.0090 \\
\hline
\end{tabular}

S. 6: $\hat{Q}_{p}$ is the empirical $p$ th quantile of $S$ in (3.6) computed from 10,000 samples when $\Delta=0.1054$. $\hat{P}\left(S>Q_{p}\right)$ is the proportion of the values of $S$ in 10,000 samples which are larger than the $p$ th quantile of a standard normal distribution. The null model $(4.1)$ has $\rho_{0}(t ; \gamma)=1, \beta=0$ and $\alpha_{i} \sim$ gamma [mean=1, variance $=0.6]$. 


\begin{tabular}{cccccccc}
\hline$m$ & $E\left\{N_{i}\left(\tau_{i}\right)\right\}$ & $\hat{Q}_{.950}$ & $\hat{Q}_{.975}$ & $\hat{Q}_{.990}$ & $\hat{P}(S>1.645)$ & $\hat{P}(S>1.960)$ & $\hat{P}(S>2.326)$ \\
\hline \multirow{2}{*}{100} & 1 & 1.630 & 1.967 & 2.337 & 0.0486 & 0.0255 & 0.0108 \\
& 2 & 1.640 & 1.941 & 2.311 & 0.0497 & 0.0240 & 0.0099 \\
& 5 & 1.657 & 1.977 & 2.316 & 0.0520 & 0.0260 & 0.0097 \\
\hline 200 & 1 & 1.635 & 1.973 & 2.321 & 0.0493 & 0.0260 & 0.0099 \\
& 2 & 1.652 & 1.940 & 2.278 & 0.0507 & 0.0236 & 0.0083 \\
& 5 & 1.669 & 1.982 & 2.312 & 0.0518 & 0.0267 & 0.0098 \\
\hline 500 & 1 & 1.616 & 1.964 & 2.269 & 0.0472 & 0.0253 & 0.0082 \\
& 2 & 1.651 & 1.956 & 2.337 & 0.0505 & 0.0247 & 0.0101 \\
& 5 & 1.635 & 1.981 & 2.341 & 0.0490 & 0.0259 & 0.0104 \\
\hline
\end{tabular}

S. 7: $\hat{Q}_{p}$ is the empirical $p$ th quantile of $S$ in (3.6) computed from 10,000 samples when $\Delta=0.2231$. $\hat{P}\left(S>Q_{p}\right)$ is the proportion of the values of $S$ in 10,000 samples which are larger than the $p$ th quantile of a standard normal distribution. The null model (4.1) has $\rho_{0}(t ; \gamma)=1, \beta=0$ and $\alpha_{i} \sim$ gamma [mean=1, variance $=0.6]$.

\begin{tabular}{ccccc|ccc|ccc}
\hline & & \multicolumn{3}{c}{$E\left\{N_{i}\left(\tau_{i}\right)\right\}=1$} & \multicolumn{3}{c}{$E\left\{N_{i}\left(\tau_{i}\right)\right\}=2$} & \multicolumn{3}{c}{$E\left\{N_{i}\left(\tau_{i}\right)\right\}=5$} \\
\cline { 2 - 10 }$\Delta$ & $\Delta_{0}$ & $e^{\beta}=1$ & $e^{\beta}=2$ & $e^{\beta}=3$ & $e^{\beta}=1$ & $e^{\beta}=2$ & $e^{\beta}=3$ & $e^{\beta}=1$ & $e^{\beta}=2$ & $e^{\beta}=3$ \\
\hline \multirow{2}{*}{0.0513} & $\frac{2}{3} \Delta$ & 0.054 & 0.343 & 0.779 & 0.055 & 0.596 & 0.972 & 0.059 & 0.954 & 1.000 \\
& $\Delta$ & 0.053 & 0.572 & 0.960 & 0.057 & 0.856 & 1.000 & 0.046 & 0.999 & 1.000 \\
& $\frac{4}{3} \Delta$ & 0.050 & 0.498 & 0.938 & 0.046 & 0.847 & 1.000 & 0.054 & 0.998 & 1.000 \\
\hline \multirow{3}{*}{0.1054} & $\frac{2}{3} \Delta$ & 0.053 & 0.491 & 0.923 & 0.049 & 0.814 & 1.000 & 0.058 & 0.996 & 1.000 \\
& $\Delta$ & 0.047 & 0.745 & 0.996 & 0.047 & 0.972 & 1.000 & 0.048 & 1.000 & 1.000 \\
& $\frac{4}{3} \Delta$ & 0.051 & 0.606 & 0.965 & 0.051 & 0.931 & 1.000 & 0.055 & 1.000 & 1.000 \\
\hline \multirow{2}{*}{0.2231} & $\frac{2}{3} \Delta$ & 0.045 & 0.542 & 0.967 & 0.048 & 0.938 & 1.000 & 0.050 & 1.000 & 1.000 \\
& $\Delta$ & 0.046 & 0.779 & 0.998 & 0.053 & 0.993 & 1.000 & 0.054 & 1.000 & 1.000 \\
& $\frac{4}{3} \Delta$ & 0.042 & 0.549 & 0.949 & 0.048 & 0.946 & 1.000 & 0.048 & 1.000 & 1.000 \\
\hline
\end{tabular}

S. 8: Proportion of times in 1,000 samples that test statistic (3.6) exceeded its 0.05 critical value for the alternative model (4.1) under various scenarios when $\phi=0.6$ and $m=100$. 


\begin{tabular}{ccccc|ccc|ccc}
\hline & & \multicolumn{3}{c}{$E\left\{N_{i}\left(\tau_{i}\right)\right\}=1$} & \multicolumn{3}{c}{$E\left\{N_{i}\left(\tau_{i}\right)\right\}=2$} & \multicolumn{3}{c}{$E\left\{N_{i}\left(\tau_{i}\right)\right\}=5$} \\
\cline { 2 - 10 }$\Delta$ & $\Delta_{0}$ & $e^{\beta}=1$ & $e^{\beta}=2$ & $e^{\beta}=3$ & $e^{\beta}=1$ & $e^{\beta}=2$ & $e^{\beta}=3$ & $e^{\beta}=1$ & $e^{\beta}=2$ & $e^{\beta}=3$ \\
\hline \multirow{3}{*}{0.0513} & $\frac{2}{3} \Delta$ & 0.039 & 0.909 & 1.000 & 0.044 & 0.997 & 1.000 & 0.057 & 1.000 & 1.000 \\
& $\Delta$ & 0.059 & 0.996 & 1.000 & 0.050 & 1.000 & 1.000 & 0.056 & 1.000 & 1.000 \\
& $\frac{4}{3} \Delta$ & 0.057 & 0.995 & 1.000 & 0.049 & 1.000 & 1.000 & 0.052 & 1.000 & 1.000 \\
\hline \multirow{3}{*}{0.1054} & $\frac{2}{3} \Delta$ & 0.050 & 0.990 & 1.000 & 0.059 & 1.000 & 1.000 & 0.047 & 1.000 & 1.000 \\
& $\Delta$ & 0.059 & 1.000 & 1.000 & 0.052 & 1.000 & 1.000 & 0.046 & 1.000 & 1.000 \\
& $\frac{4}{3} \Delta$ & 0.054 & 1.000 & 1.000 & 0.047 & 1.000 & 1.000 & 0.056 & 1.000 & 1.000 \\
\hline \multirow{2}{*}{0.2231} & $\frac{2}{3} \Delta$ & 0.046 & 1.000 & 1.000 & 0.048 & 1.000 & 1.000 & 0.049 & 1.000 & 1.000 \\
& $\Delta$ & 0.038 & 1.000 & 1.000 & 0.055 & 1.000 & 1.000 & 0.052 & 1.000 & 1.000 \\
& $\frac{4}{3} \Delta$ & 0.049 & 0.997 & 1.000 & 0.063 & 1.000 & 1.000 & 0.061 & 1.000 & 1.000 \\
\hline
\end{tabular}

S. 9: Proportion of times in 1,000 samples that test statistic (3.6) exceeded its 0.05 critical value for the alternative model (4.1) under various scenarios when $\phi=0.6$ and $m=500$.

\section{A simulation study for the misspecification of gamma random effect}

Details of the simulation study in this section are given in the last paragraph of Section 4 . In the following table, the $\alpha_{i}$ were generated from a lognormal distribution with mean 1 and variance $\phi$. Empirical Type 1 error and power results are based on 1,000 realizations of $m$ processes.

\begin{tabular}{lccccccccc}
\hline & \multicolumn{4}{c}{$m=20, \tau=10$} & & \multicolumn{4}{c}{$m=40, \tau=10$} \\
\cline { 2 - 4 } \cline { 8 - 10 }$\phi$ & $e^{\beta}=1$ & $e^{\beta}=2$ & $e^{\beta}=3$ & $e^{\beta}=4$ & & $e^{\beta}=1$ & $e^{\beta}=2$ & $e^{\beta}=3$ & $e^{\beta}=4$ \\
\hline 0.3 & 0.047 & 0.499 & 0.904 & 0.989 & & 0.051 & 0.774 & 0.990 & 0.999 \\
0.6 & 0.049 & 0.556 & 0.908 & 0.988 & & 0.060 & 0.805 & 0.994 & 1.000 \\
\hline
\end{tabular}

S. 10: Empirical Type 1 error and power of the test statistic (3.6) with respect to the misspecification of $\alpha_{i} ; \Delta=0.0202$. 